\title{
Le touriste consommateur : un caméléon...
}

\section{Alain Decrop}

\section{OpenEdition}

\section{Journals}

Édition électronique

URL : http://journals.openedition.org/tourisme/505

DOI : 10.4000/tourisme.505

ISSN : 2492-7503

\section{Éditeur}

Éditions touristiques européennes

\section{Édition imprimée}

Date de publication : 1 juin 2011

Pagination : 4-13

ISSN : 2109-5671

\section{Référence électronique}

Alain Decrop, « Le touriste consommateur : un caméléon... », Mondes du Tourisme [En ligne], 3 | 2011, mis en ligne le 30 septembre 2015, consulté le 19 avril 2019. URL : http://journals.openedition.org/ tourisme/505; DOI : 10.4000/tourisme.505

\section{(c)}

Mondes du tourisme est mis à disposition selon les termes de la licence Creative Commons Attribution - Pas d'Utilisation Commerciale - Pas de Modification 4.0 International. 


\section{Le touriste consommateur : un caméléon...}

\section{Alain DECROP} [alain.decrop@fundp.ac.be]

Professeur de marketing, Facultés universitaires Notre-Dame de la Paix, Namur Directeur du Center for Research on Consumers and Marketing Strategy (Louvain School of Management)

Résumé. Cet article s'attache à celui qui constitue la raison d'être de l'activité touristique, à savoir le "touriste consommateur". Après avoir introduit les paradigmes envisageables pour étudier les comportements touristiques, nous analysons les processus de décision qui les sous-tendent et les variables (personnelles, interpersonnelles et environnementales) qui les influencent, avant de terminer par l'exposé d'une nouvelle typologie des vacanciers fondée sur leurs processus de prise de décision. L'article montre que le touriste consommateur actuel cause bien des soucis au gestionnaire et au "marketeur". Loin de se comporter de manière rationnelle et prévisible, il échappe de plus en plus aux modèles et aux segmentations classiques. La transition de l'époque industrielle (moderne) à l'ère des services et de l'information (postmoderne) laisse des traces indélébiles sur les comportements, les valeurs et les styles de vie. Les deux dernières décennies ont ainsi marqué l'avènement d'un touriste caméléon et insatiable.
Cet article reprend de larges extraits de : Alain DECROP, Le Touriste consommateur, De Boeck, 2010. Reproduit avec l'aimable autorisation de l'éditeur.

Abstract. This paper focuses on tourist consumers who lie at the core of the tourist industry. We first introduce the major paradigms that may be used to investigate tourist behaviors; we then analyse the decision processes which underlie them as well as the (personal, interpersonal and environmental) variables which influence them. Finally, we present a new typology of vacationers based on their decision-making processes. The paper indicates that managers and marketers may be embarrassed by contemporary tourist consumers. Far from being rational and predictable, these go beyond traditional models and segmentations. The transition from the industrial (modern) epoch to the (postmodern) era of information and services leaves indelible marks on behaviours, values and life styles. The last two decades have paved the way for the emergence of a chameleon insatiable tourist. 
$\mathbf{L}$ e comportement du consommateur s'intéresse aux activités impliquées dans l'obtention, la consommation et l'élimination des produits ou services, y compris les processus de décision et de consommation qui précèdent ou suivent ces activités (Blackwell, Miniard et Engel, 2006). Que l'on soit entreprise privée, service public ou citoyen, il est tout aussi important de s'intéresser aux comportements du touriste consommateur. Pour les entreprises actives dans le domaine du tourisme et des loisirs, il est évidemment fondamental de bien comprendre les besoins et désirs de leurs clients cibles, étant donné que c'est la satisfaction de ces besoins et désirs qui fonde l'optique marketing ou l'orientation marché. En d'autres mots, il est difficile de concevoir, de communiquer et de vendre un produit si celui-ci ne correspond pas aux attentes des consommateurs. Pour les institutions publiques, il est également important de comprendre le touriste consommateur, afin notamment de répondre efficacement aux demandes de ses citoyens et de mettre en place des politiques et des lois adéquates pour les informer ou les protéger. Enfin, comprendre les comportements peut également s'avérer utile pour les touristes consommateurs eux-mêmes, pour les aider à prendre des décisions efficaces et efficientes ou les rendre plus critiques par rapport aux sources commerciales ou non commerciales susceptibles d'influencer leurs comportements.

Dans cet article, nous allons précisément résumer les principes des comportements et de la prise de décision du vacancier, ainsi que leurs implications pour l'entreprise et le marketing. Mais avant cela, il s'agit de présenter les principaux paradigmes qui peuvent être utilisés pour étudier décisions et comportements touristiques.

\section{PARAdigMeS D'ÉTUde DES COMPORTEMENTS}

D'un point de vue fondamental, cinq grandes approches théoriques peuvent être mobilisées pour mieux comprendre les décisions et les comportements du touriste consommateur : l'approche microéconomique, l'approche motivationnelle, l'approche behavioriste, l'approche cognitiviste et l'approche postmoderne.

La micro-économie propose une approche normative des comportements avec la vision d'un consommateur rationnel, qui prend des décisions afin de maximiser son utilité (sa satisfaction) sous contrainte de son budget. Cette approche est holiste, statique et purement individuelle : les produits sont présentés comme des entités globales, les décisions sont considérées comme intemporelles et détachées de toute influence environnementale.

L'approche motivationnelle, ins- pirée des réflexions psychanalytiques de Dichter, Freud ou Jung, s'intéresse moins aux résultats (utilité) des comportements qu'à ses causes subjectives. L'objectif est de comprendre les raisons profondes qui sous-tendent les comportements d'achat. Les chercheurs en psychanalyse estiment que ces raisons sont souvent cachées ou inavouées et qu'il faut donc pénétrer le monde intérieur de l'individu, celui du vécu et des expériences antérieures, pour les découvrir.

La recherche behavioriste partage avec l'approche motivationnelle la même volonté de comprendre les raisons des comportements pour mieux motiver les consommateurs à rester fidèles à l'entreprise. Toutefois, le behaviorisme propose une vision déterministe, postulant qu'il est possible de conditionner les individus pour produire des réponses mécaniques. Ainsi, la répétition de stimuli publicitaires permettrait de conduire à des habitudes d'achat fermes en provoquant puis en renforçant des réponses positives chez le consommateur.

Le courant cognitiviste représente aujourd'hui l'approche dominante en comportement du consommateur. Largement inspirée par la psychologie cognitive et sociale, cette approche donne la primauté au monde mental (cognitif) de l'individu ; l'objectif étant de comprendre le cheminement suivi par le consommateur pour 
Figure | • Niveaux dans la prise de décision de vacances

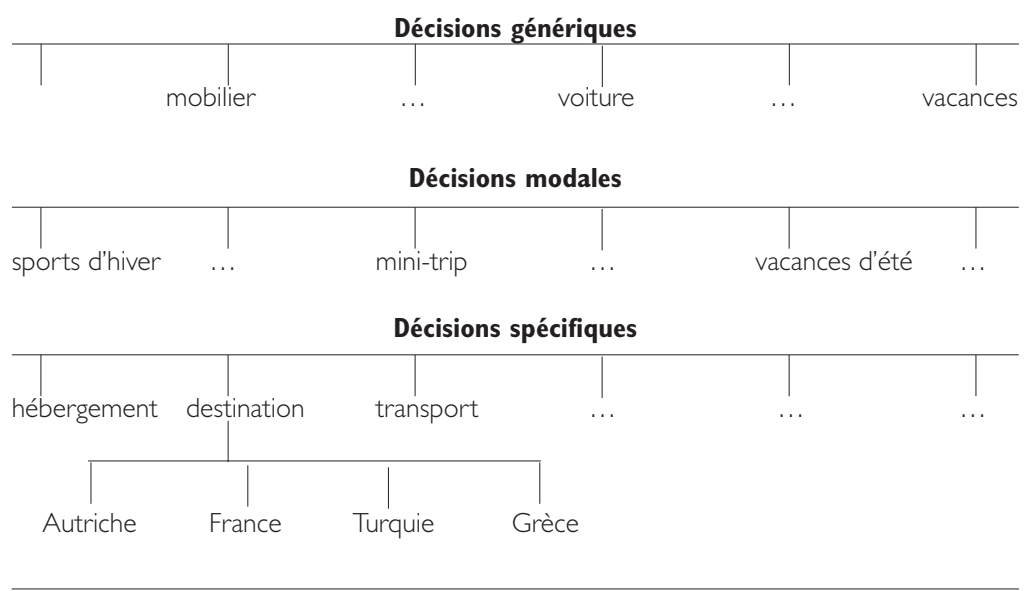

résoudre ses problèmes. Contrairement aux autres approches, le courant cognitiviste propose la vision d'un consommateur acteur, et non pas spectateur, de ses décisions et de ses comportements ; le processus perceptuel et l'environnement social en constituent les pierres angulaires. Le consommateur est présenté comme un être rétif au risque, qui n'aura de cesse de collecter et de traiter de l'information pour aboutir à des choix qui le satisfont.

Enfin, plus récemment, a émergé le courant postmoderne, qui est marqué par une ouverture de l'étude du comportement des consommateurs aux niveaux ontologique, épistémologique et méthodologique. Le postmodernisme ne nie pas l'intérêt des approches antérieures, notamment en matière de traitement de l'information, mais considère leurs apports sous un angle moins normatif et plus rela- tif, parce que les chercheurs se rendent compte que le consommateur est le plus souvent confronté à des choix routiniers et qu'il se laisse guidé par des règles de décision simples (par exemple : "J'achète le produit le moins cher"). Le contexte et, en particulier, la dimension culturelle et le niveau d'implication du consommateur sont pris en compte pour mieux comprendre les différences comportementales entre individus et entre situations de choix. Enfin, l'approche postmoderne s'ouvre à des centres d'intérêt délaissés jusqu'ici par les chercheurs en marketing, tels que les dimensions affectives des choix et les aspects expérientiels de la consommation (amusement, sensations, fantasmes, etc.). De même, un regard nouveau est posé sur la possession et la consommation en tant que tels et sur le sens que ces processus donnent à la vie de l'individu.
Dans les sections qui suivent, c'est surtout dans les approches cognitives et postmodernes que nous allons puiser pour essayer de comprendre comment le touriste se comporte et, plus particulièrement, comment il prend ses décisions d'achat. Nous n'envisagerons pas ici les expériences de consommation qui font l'objet d'un long développement dans le livre de Decrop (2010).

\section{DÉCISIONS ET COMPORTEMENTS TOURISTIQUES}

Lorsqu'ils consultent un guide, discutent de leur prochaine destination lors du repas familial, se rendent à l'agence de voyages ou bouclent leurs valises, peu de vacanciers se rendent compte qu'ils sont engagés dans un processus de décision. Celui-ci peut s'avérer plus ou moins complexe selon les individus et les situations. Quand nous partons en vacances, nous sommes amenés à prendre toute une série de décisions : Quand partir ? Quelle destination choisir ? Comment y aller ? Où dormir ? Dans la section suivante, nous proposons une nomenclature complète de ces décisions, avant de détailler la manière dont les touristes prennent leurs décisions grâce à deux références principales, le modèle de Van Raaij et Francken (1984, pp. I0 I- | | 2) ainsi que notre propre conceptualisation (Decrop, 2006). Ensuite, nous examinerons les principaux fac- 
teurs qui influencent les décisions et comportements touristiques, avant de terminer par l'exposé d'une nouvelle typologie des vacanciers, établie sur leurs processus de prise de décision

\section{Nomenclature}

\section{des décisions de vacances}

Au contraire de bien d'autres produits et services, les vacances et les voyages peuvent être considérés comme des domaines de décision complexes, en ce sens qu'ils incluent de nombreuses sous-décisions et de multiples choix à opérer. Pour éviter de tomber dans le travers de certains modèles de prise de décision qui ne spécifient pas leur objet (les vacances ? la destination ?), il est important de définir toutes les décisions qui peuvent être impliquées dans les vacances et les voyages.

Decrop et Kozak (2006) opèrent une distinction entre trois niveaux de décision : générique, modal et spécifique ( $c f$. figure 1 ). Le niveau de la décision générique de partir ou ne pas partir en vacances demande au consommateur d'effectuer des arbitrages entre alternatives de choix non comparables (Johnson, 1984, pp. 74I-753), comme partir en vacances ou acheter du nouveau mobilier. Au contraire, les décisions spécifiques portent sur des alternatives comparables, qui sont représentées par les mêmes attributs. Par exemple, le touriste choisit entre trois hôtels caractérisés par une localisation, un prix et un niveau du confort. Le niveau modal concerne les choix de mode, ou type, de vacances : des vacances d'été ou des sports d'hiver ? Des vacances à la mer ou à la montagne ? Un séjour à un seul endroit ou un circuit itinérant ? Ce niveau modal se situe à mi-chemin entre le niveau générique et le niveau spécifique, puisque certaines sousdécisions sont considérées quel que soit le type de vacances (par exemple, les questions de la destination, de l'hébergement et du transport se posent aussi bien pour les vacances d'été que pour les sports d'hiver), tandis que d'autres sont spécifiques à un seul type (par exemple, le matériel de ski) et impliquent donc des alternatives de choix non comparables avec les autres modes de vacances.

En ce qui concerne les décisions spécifiques, on peut distinguer quinze éléments susceptibles d'être considérés dans le processus de décision de vacances ( $c f$. figure 2$)$ : l'accompagnement, la destination, le mode de transport, l'hébergement, la période, la durée, les achats, l'itinéraire pour se rendre à la destination, les activités, le mode d'organisation, les attractions à visiter, le budget et la nature des dépenses, les choix de repas, le circuit de visite (sur place) et le style de vacances.

\section{Processus de décision}

De nombreux modèles ont été développés dans la littérature pour expliquer la prise de décision tou- ristique. Parmi les plus populaires, citons les modèles de Van Raaij et Francken (1984, pp. 10 I-1 I2), de Moutinho (1987, pp. 2-44), de Woodside et Lysonski (1989, pp. 814) et de Woodside et MacDonald (1994, pp. 30-59). Largement influencés par l'œuvre d'Engel et Blackwell (1982), Van Raaij et Francken (1984) décrivent ainsi un processus de décision en cinq étapes.

1) La décision générique de partir : vais-je partir ou non en vacances? Ce choix dépend largement du revenu du ménage mais aussi de la situation familiale, de la possession d'une infrastructure de vacances (caravane, maison de vacances, etc.), du style de vie et des valeurs du vacancier. Dans la plupart des cas, la décision est prise en groupe (avec le mari, la femme et même les enfants). On peut également noter qu'il existe plusieurs stratégies pour aboutir à cette décision : le compromis, la négociation et le marchandage.

2) L'acquisition d'informations. La recherche d'informations dépend fortement des caractéristiques de l'individu. Elle peut servir à différentes choses : convaincre une personne de partir en vacances, influencer son choix particulier de destinations (ou celui de quelqu'un d'autre) ou encore rationaliser, justifier ou légitimer une décision (dans ce cas, l'information dite négative est importante). Différentes sources d'informations peuvent être utilisées, parmi lesquelles on distingue les sources 


\section{Figure 2 - Schéma global de la prise de décision de vacances}

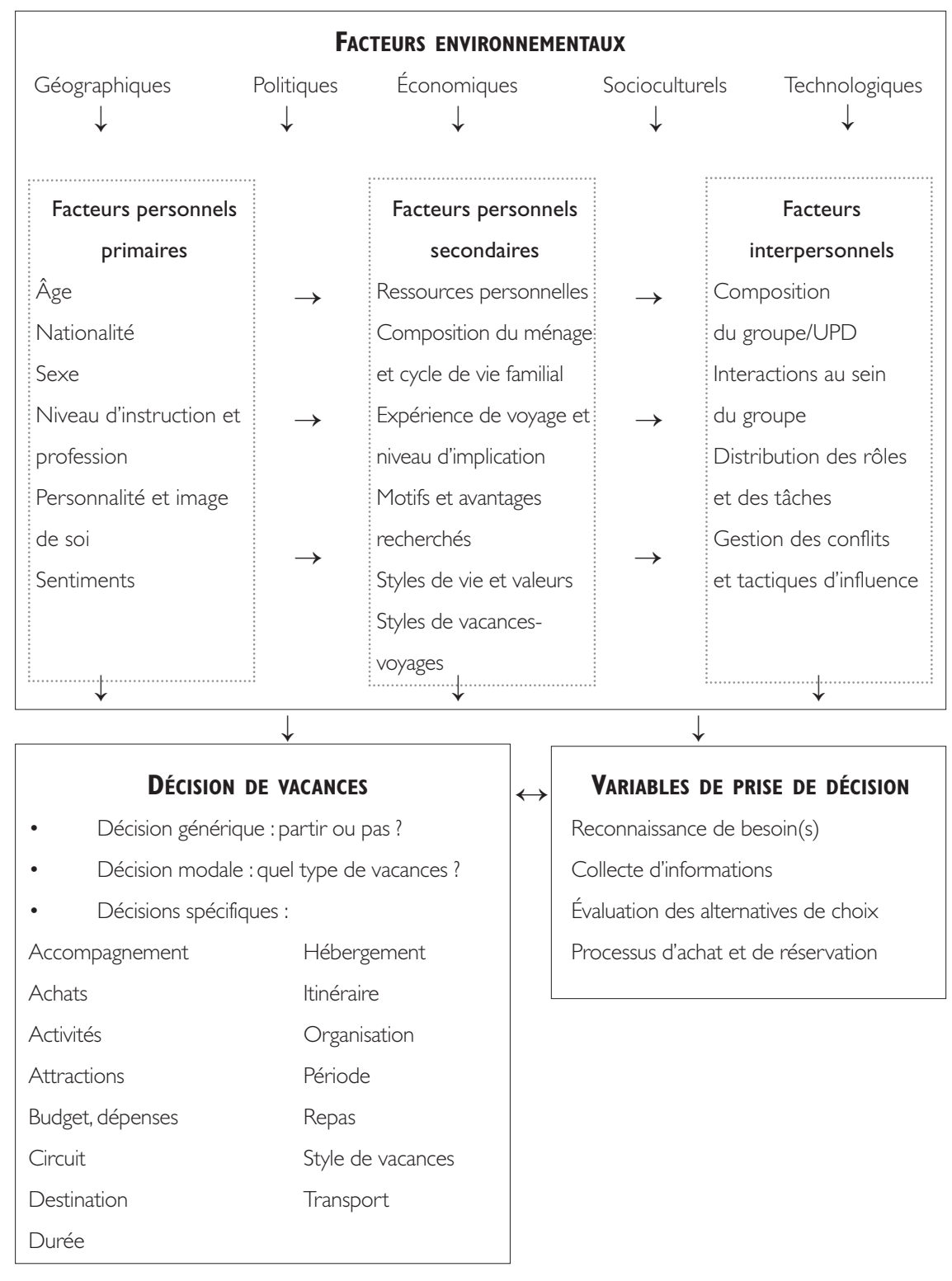

commerciales (brochures et catalogues, publicités dans les médias, sites web des prestataires, agents de voyages, etc.) des sources non commerciales (parents, amis, collègues, autres consommateurs, etc.). 3) La prise de décision jointe. Elle consiste à choisir une alternative de vacances avec les différents membres du ménage. Van Raaij et Francken (1984, pp. I0 I- I | 2) identifient plusieurs déterminants du processus de prise de décision : la publicité des agences de voyages, la communication et le style d'interaction au sein du ménage, le niveau d'éducation et l'expérience de vacances, la sensibilité au prix et à d'autres attributs des vacances, la fidélité à certaines destinations et les types de vacances. Les auteurs précisent encore que pour certains touristes, la prise de décision est un long processus de recherche d'informations et de comparaison des alternatives de choix tandis que pour d'autres, elle peut se résumer à une décision impulsive de dernière minute.

4) L'expérience de vacances. Celleci implique toute une série d'activités, souvent révélatrices des préférences des touristes, qui sont vécues pendant le voyage en tant que tel. Meyer (1977, pp. 259-29 I) distingue sept types d'activités des vacanciers : l'aventure (découverte et innovation, mais avec peu de confort matériel), l'expérience (de nouvelles expériences, mais sans le côté peu confortable de l'aventure), la conformité (activités habituelles, semblables à celles qui sont pratiquées à la maison), l'éducation (activités culturelles, historiques...), la santé (repos et confort), le contact social (établir un contact avec des personnes inconnues, se faire de nouveaux amis...), le statut (établir des contacts sociaux avec des personnes de la même classe sociale). 5) Le niveau de satisfaction postvacances. Il s'agit de la différence entre ce que l'on espérait et ce que l'on a effectivement reçu. Le tou- 
riste sera satisfait si ses vacances sont conformes ou meilleures que ce qu'il imaginait ; dans le cas contraire, il sera mécontent. Van Raaij et Francken (1984, pp. I0II I2) postulent également que l'attribution du mécontentement déterminera le comportement de plainte éventuel du client. Les vacanciers attribuant leur mécontentement à des facteurs externes (de mauvais conseils de l'agence de voyages, par exemple) seront davantage mécontents et se plaindront plus que les vacanciers qui imputent leur mécontentement à eux-mêmes (attribution interne). Cependant, on constate une tendance naturelle des consommateurs à attribuer leur mécontentement à des facteurs externes et à justifier leur satisfaction par des facteurs internes.

Nous avons souhaité apporter un éclairage plus contextuel et "naturaliste" que ces modèles trop déshumanisés, rationnels et détachés de la réalité, grâce à une étude qualitative des processus de décision des vacanciers (Decrop, 2006). Ainsi, pendant un an, nous avons interviewé vingt-cinq ménages belges (single, couples, familles, groupes d'amis) à plusieurs reprises pour pouvoir suivre l'évolution de leurs plans et décisions de vacances d'été. Nous avons également réalisé des sessions d'observation au Salon des vacances de Bruxelles et dans deux agences de voyages. Parmi les principaux résultats de l'étude, il apparaît que la prise de décision de vacances est souvent déclenchée incidemment, à travers des conversations, une émission à la télévision, une brochure reçue ou l'invitation de parents ou amis. Il y a rarement une évolution linéaire (c'est-à-dire séquentielle et hiérarchique) des plans de vacances. Les voyages et les produits touristiques ne font pas toujours l'objet d'une forte implication du consommateur; il arrive que certains ne soient pas intéressés et que d'autres voient leur implication dans la prise de décision limitée par la routine ou l'intervention de contraintes. Ce manque d'implication et ces contraintes sont responsables de beaucoup de changements et de retournements dans les plans de vacances.

Les facteurs émotionnels sont particulièrement puissants dans le façonnement des choix de vacances. Il arrive ainsi que l'on prenne une décision de manière impulsive sur la base d'émotions ou d'humeurs ressenties. Nos données indiquent en effet que la prise de décision de vacances est empreinte de sensations, de rêverie, de nostalgie ou d'anticipation. Ainsi, le "mode de choix affectif" (Mittal, 1988, pp. 499-524) peut s'avérer tout aussi pertinent que les paradigmes traditionnels de résolution de problème et de traitement de l'information (Bettman, 1979) pour expliquer le processus de décision touristique. La résolution de problème n'est pas aussi étendue et la recherche d'informations n'est pas aussi intense que cela est généralement présenté dans la littérature.

Ce n'est pas pour autant que la planification de vacances est totalement déstructurée. Souvent, en effet, on observe une certaine évolution dans la prise de décision. Ainsi, les touristes ont tendance à être optimistes et idéalistes au début de leurs plans de vacances. Ils deviennent plus réalistes avec le temps, au fur et à mesure que la période de vacances approche et que l'engagement à choisir devient plus pressant. Cette évolution s'explique essentiellement par l'intervention de facteurs contextuels : les facilitateurs contextuels (adjuvants) sont considérés en premier, tandis que les inhibiteurs contextuels (contraintes) sont pris en considération plus tard (Um, 1990, pp. 7I I). L'intervention de ces variables situationnelles et sociales oblige souvent les vacanciers à adapter leurs décisions de vacances en fonction des opportunités (Payne et al., 1993).

En conclusion, on peut dire que la planification de vacances est un processus continu emprunt d'adaptabilité et d'opportunisme où les émotions, l'imagination et les contraintes jouent un rôle important. Cette adaptabilité et cet opportunisme ont un impact majeur sur le timing des décisions, la recherche d'informations et la prévisibilité des choix. 


\section{Facteurs d'influence}

des décisions et comportements

Il existe une multitude de facteurs qui influencent les décisions et la prise de décision de vacances. Nous pouvons regrouper ces facteurs en trois grands types : les facteurs personnels, les facteurs interpersonnels et les facteurs environnementaux. Comme le suggère la figure 2, ces facteurs opèrent dans une certaine logique. Les facteurs environnementaux, tels que la culture, le cadre géographique ou le contexte politique, sont des éléments structurels sur lesquels le vacancier n'a pas de prise directe. Ils englobent tous les autres types de facteurs. Ensuite, on peut opérer une distinction entre facteurs personnels primaires et secondaires. L’âge, la nationalité, le sexe, le niveau d'instruction et la profession, la personnalité, l'image de soi et les sentiments sont les racines individuelles dans lesquelles viennent se greffer les autres facteurs personnels (expérience de vacances, ressources personnelles, motifs, implication, etc.). Les facteurs personnels s'imbriquent naturellement dans la prise de décision de groupe, étant donné que les décisions de vacances impliquent des décisions jointes. La composition du groupe, les interactions en son sein, la distribution des rôles et la gestion des conflits dans le groupe sont autant de facteurs interpersonnels qui orientent jugements et décisions de vacances.

La plupart des variables reprises dans la figure 2 peuvent avoir tantôt une influence favorable ou facilitatrice sur les choix touristiques, tantôt un impact négatif ou inhibiteur sur ces choix. Par exemple, les ressources disponibles, l'état de santé du touriste ou les interactions au sein du groupe peuvent ainsi intervenir pour faciliter ou, au contraire, freiner une décision de vacances. De la même manière, ces variables peuvent avoir une influence permanente/structurelle ou momentanée/situationnelle sur la prise de décision. Les influences structurelles renvoient au contexte général et aux conditions qui ont un impact ou sont susceptibles d'influencer les comportements touristiques de manière durable et continue, tandis que les influences situationnelles concernent des conditions, propres à un moment et à un lieu donnés, qui influencent ces comportements ou les ont influencés ponctuellement, de manière temporaire (Belk, 1975, pp. 157-167). Par exemple, la situation économique et la profession ont une influence structurelle, tandis que le budget disponible à un moment donné et le fait (pour un étudiant) d'avoir un job de vacances cet été-là exercent une influence situationnelle. De la même manière, le climat peut avoir à la fois un impact structurel et/ou situationnel. D'une part, pour un vacancier belge ou du nord de la France, le climat, pas très clément sous ces cieux, a une influence permanente sur la décision de vacances et sur les préférences pour les destinations ensoleillées du sud de l'Europe. D'autre part, de mauvaises conditions climatiques (par exemple, s'il pleut depuis quinze jours) peuvent avoir un impact négatif sur l'humeur du consommateur et accroître son désir de partir en vacances et/ou de choisir une destination ensoleillée cet été-là.

\section{Une typologie des touristes \\ fondée sur leur processus \\ de décision}

Grâce à l'étude empirique décrite plus haut, nous avons pu établir une nouvelle typologie de vacanciers. Notre typologie ne repose plus sur les critères démographiques ou socio-économiques classiques, mais sur des variables psychologiques et comportementales plus fondamentales. Six types de vacanciers peuvent ainsi être décrits : le routinier, le rationnel, l'hédoniste, l'opportuniste, le contraint et l'adaptable (pour plus de détails, voir Decrop et Snelders, 2005, pp. 121-132).

Le vacancier routinier n'est pas vraiment impliqué par son processus de décision, étant donné qu'il reproduit chaque année les mêmes comportements de vacances. C'est souvent sa personnalité (il est plutôt passif et casanier) ou son contexte (il possède une seconde résidence en France) qui le conduit à l'habitude. À beaucoup d'égards, ce type de vacancier déteste le risque : il 
ne veut pas gaspiller son temps à découvrir un nouvel environnement et aime se sentir chez lui une fois arrivé à destination. Sa préparation du voyage se limite donc souvent au simple fait de trouver de nouvelles activités ou excursions à faire dans une région qui lui est familière.

L'aversion du risque influence également la planification et les décisions du vacancier rationnel. Il n'est pas prêt à partir à n'importe quel prix et reste souvent fidèle à des repères qui le rassurent (par exemple, la même chaîne d'hôtels). Prudent et réaliste, le vacancier rationnel commence tôt à réfléchir à ses vacances d'été (janvier-février) ; il fait preuve de beaucoup de minutie dans la préparation et prend ses décisions dès que possible. Tout doit être planifié avant de partir pour qu'il ne manque pas des aspects importants du voyage (tel monument ou tel musée) ou qu'il évite de mauvaises surprises (plus de chambre libre). C'est pourquoi il recueille plus d'informations que les autres types de vacanciers.

Tout comme le rationnel, le vacancier hédoniste est fortement impliqué par ses voyages. Cependant, c'est la stimulation émotionnelle et non plus l'aversion du risque qui est le moteur de son implication. C'est ainsi que l'hédoniste prend beaucoup de plaisir à penser à ses vacances, à en rêver ou à en parler. Il saisit toutes les occasions pour collec- ter de l'information touristique : une émission télévisée, une brochure, une visite chez des amis. En outre, il n'hésitera pas à pousser son entourage à partir et à lui recommander des destinations particulières. De nature optimiste, le vacancier hédoniste néglige souvent les contraintes, ce qui l'amène à revoir tardivement ses projets à la baisse : il rêvait de s'envoler vers Rio, il se contentera de la mer du Nord...

Au contraire de l'hédoniste, le vacancier opportuniste ne pense pas beaucoup à ses vacances. Soit par manque d'intérêt, parce qu'il est trop occupé professionnellement pour cela, ou parce qu'il préfère saisir l'occasion quand elle se présente. Ainsi, il peut volontairement attendre une opportunité sociale (l'invitation d'un ami) ou financière (une offre de dernière minute) avant de se décider. Imprévisible et attentiste, le vacancier opportuniste minimise la planification et la préparation de ses vacances. Ses décisions surgissent de la simple coïncidence temporelle d'un besoin ("Je me sens déprimé par la grisaille actuelle") et d'une solution de choix ("Je viens de voir une offre alléchante pour le Maroc sur Liberty TV").

Notre avant-dernier type, le vacancier contraint, subit les décisions davantage qu'il ne les mầtrise. Ses contraintes peuvent être de trois ordres : d'ordre personnel (problèmes de santé), situationnel (manque d'argent) ou inter- personnel. Dans une famille, par exemple, les enfants peuvent être contraints par leurs parents de partir à la montagne alors qu'ils auraient choisi la mer. De même, des membres d'un groupe d'amis peuvent avoir des difficultés à se mettre d'accord en raison de préférences individuelles divergentes. Du fait de ces conflits, certains vacanciers finissent par partir en vacances à contrecœur, forcés par d'autres à voyager, à faire telle activité ou à visiter tel endroit.

À l'opposé du vacancier contraint, le vacancier adaptable aime voyager. Il multiplie les formules de voyage et les modes d'accompagnement : il part tantôt en famille, tantôt avec des amis, tantôt en couple pour un week-end en amoureux. Le vacancier adaptable a toujours plusieurs projets en tête, qu'il se met un point d'honneur à faire aboutir, en adaptant choix et mode de décision à la situation. La flexibilité est également de mise pendant le séjour luimême : "Ce n'est parce qu'on avait prévu de faire telle activité qu'on la fera nécessairement...”, entendon dire. Évidemment, ces adaptations conduisent souvent à des choix peu prévisibles et tardifs.

\section{LE TOURISTE CONSOMMATEUR, UN CAMÉLÉON...}

La typologie que nous venons de présenter indique à suffisance qu'il n'y a pas une seule mais plu- 


\section{Figure $3 \cdot$ Le touriste en transition}

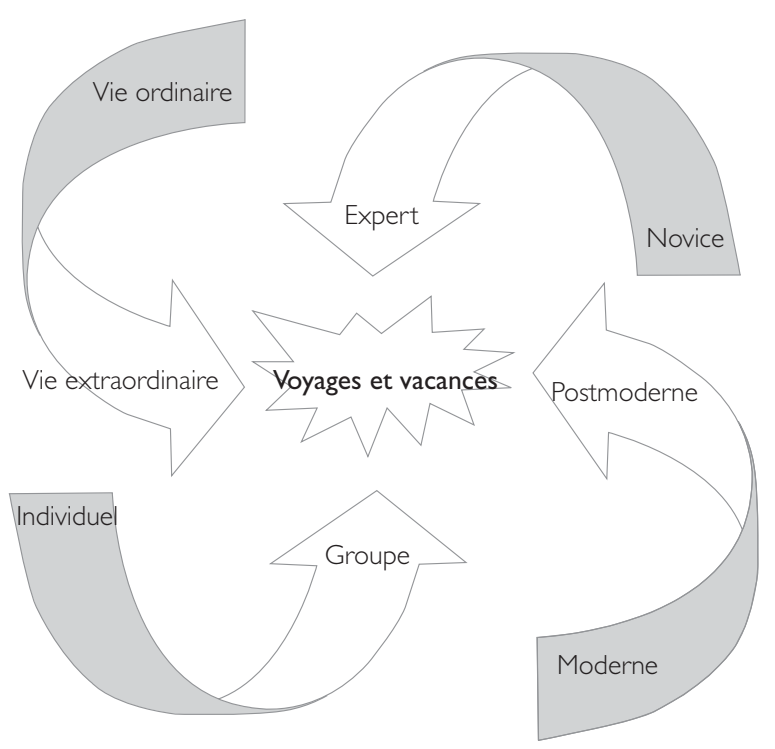

sieurs manières de prendre ses décisions de vacances, en fonction de l'individu, du groupe dans lequel il s'inscrit et de la situation dans laquelle il se trouve. De manière plus générale, le thème de la transition (Decrop, 2000, pp. 169-179) peut être utilisé comme reflet synthétique de l'impact de tout ce contexte personnel, interpersonnel et environnemental qui influence le vacancier dans ses décisions. Par transition, nous entendons "le passage d'un état de choses à un autre". Quand il part en vacances, l'être humain s'engage vers l'au-delà. Il s'engage d'abord en dehors de sa vie (ordinaire) de tous les jours pour une autre vie (extraordinaire). Il s'engage ensuite dans un processus de socialisation : il communique ses valeurs et préférences personnelles au groupe au sein duquel il prend ses décisions. En outre, le vacancier s'engage dans une sorte de "carrière" qui le voit "grandir" au fur et à mesure qu'il acquiert expérience et maturité : de novice, il devient expert... Enfin, le touriste actuel s'inscrit pleinement dans la postmodernité : éclectique et insatiable, il recherche des expériences sans cesse renouvelées. Ces quatre dimensions transitionnelles sont illustrées par la figure 3.

Ainsi, le vacancier postmoderne fait preuve de désirs et de préférences sans cesse renouvelés et plus complexes. Cette mouvance et cette complexité s'inscrivent dans les mutations socio-économiques qui touchent le tourisme d'aujourd'hui et affectent celui de demain. Les professionnels du tourisme doivent s'inspirer de ces différentes dimensions transitionnelles décrites dans la figure 3. Il faut proposer au vacancier des produits qui l'enthousiasment et lui permettent de s'évader de la vie monotone de tous les jours. Les tour-opérateurs devraient davantage se mettre à l'écoute des souhaits de l'unité de prise de décision, qu'elle soit couple, famille ou groupe d'amis, et pas seulement des besoins individuels. En outre, il s'agit d'accorder une attention soutenue à l'expérience et à la maturité croissantes des vacanciers actuels dès lors qu'elles induisent une spirale "incrémentielle" de leur implication et de leur motivation. Enfin, le touriste postmoderne ne veut plus se contenter d'un rôle passif de consommateur, mais souhaite devenir producteur ou "consom'acteur" de ses choix et de ses comportements. Il désire s'impliquer davantage dans la création de ses produits touristiques (on parle à cet égard de co-création de valeur) ou dans ses pratiques de voyage (on qualifie souvent cet état d'esprit de tourisme participatif). Ainsi, les tour-opérateurs doivent-ils tout autant permettre au voyageur de développer ses séjours à la carte en interactivité (via internet, le web 2.0 , etc.) que des projets rapides clé en main. De manière générale, les prestataires touristiques doivent éviter les cloisonnements et les ciblages trop hermétiques parce que, plus que jamais, le touriste consommateur est devenu caméléon et omnivore. Quel animal... 


\section{RÉFÉRENCES BIBLIOGRAPHIQUES}

Russel W. BeLK, "Situational variables and consumer behaviour", Journal of Consumer Research, 2, 2, 1975.

James R. BetTMAN, An Information Processing Theory of Consumer Choice, Addison Wesley, 1979.

Roger D. BLACKWELL, Paul W. MiniARD et James F. ENGEL, Consumer Behavior, Thomson South-Western, 10éd., 2006.

Alain DeCROP, "Le vacancier en transition", Revue belge de géographie, 123, 2000.

Alain DeCrop, Vacation Decision Making, Cabi, 2006.

Alain DECROP, Le Touriste consommateur. Comprendre les comportements pour améliorer son efficacité marketing, De Boeck, 2010.

Alain DeCrop et Metin KozAK, "A multi-level framework for studying vacation decision making", Actes de la 35e conférence EMAC, 2006.

Alain DeCROP et Dirk SNeLDERS, "A grounded typology of vacation decision making", Tourism Management, 26, 2005.

James F. Engel et Roger D. BLACKWELL, Consumer Behavior, The Dryden Press, 1982. Michael D. JOHNSON, "Consumer choice strategies for comparing noncomparable alternatives", Journal of Consumer Research, I I, 3, 1984.

Wolfgang MeYer, "Aktivität im Urlaub", dans Klaus Dieter HARTMANN et Karl Fritz KoEPPLER (dir.), Fortschritte der Marktpsychologie, Fachbuchhandlung der Psychologie, 1977.

Banwari MITTAL, "The role of affective choice mode in the consumer purchase of expressive products", Journal of Economic Psychology, 9, 1988.

Luiz MoutINHO, "Consumer behaviour in tourism", European Journal of Marketing, 21, I0, 1987.

John W. PAYNe, James R. Bettman et Eric J. Johnson, The Adaptive Decision Maker,

Cambridge University Press, 1993.

Seoho UM, "The roles of perceived inhibitors and perceived facilitators in the pleasure travel destination choice process", dans The Tourism Connection : Linking Research and Marketing, Actes de la $21^{\text {e }}$ conférence de Travel and Tourism Research Association, 1990.

W. Fred Van RaAl] et Dick A. Francken, "Vacations decisions, activities and satisfaction", Annals of Tourism Research, II, 1984.

Arch G. WOODSIDE et Steven LYSONSKI, "A general model of traveler destination choice", Journal of Travel Research, 27, 1989.

Arch G. WOODSIDE et Roberta MACDONALD, "General system framework of customer choice processes of tourism services", dans Ruth V. GASSER et Klaus WeIERMAIR (dir.), Spoilt for Choice. Decision Making Processes and Preference Change of Tourists : Intertemporal and Intercountry Perspectives, Kulturverlag, 1994. 